\title{
Dynamic Stress Analysis of a Composite Electromagnetic Track
}

\author{
Z. G. Tian, ${ }^{1}$ X. Y. An, Y. Yang, and L. K. Zhao
}

Key Laboratory of Mechanical Reliability for Heavy Equipments and Large Structures of Hebei Province, Yanshan University, Qinhuangdao, China

1tianzhenguo1@163.com

The firing of an electromagnetic gun is shown to result in the armature moving along the guide rail, which can cause extrusion, wear, planing and other problems, which set limits on its application. The track properties can be adjusted by changing its composition, so as to obtain good electrical conductivity, corrosive resistance, and strength. For simplicity, the composite track is presented as an elastic foundation beam, and the general solution of composite track deflection at dynamic load is obtained by using the two-dimensional Fourier integral transformation, on this basis, the bending moment distribution and expression for dynamic stress are obtained. The characteristics of dynamic stress distribution and factors that influence them are analyzed, the effect of the proportion of the composite layer and its parameters on the dynamic stress of the track is discussed.

Keywords: composite track, dynamic stress, Fourier transform, dynamic load, elastic foundation beam.

Introduction. The electromagnetic gun can obtain higher speed than the traditional launching mode [1] and can save energy and reduce reaction time. However, some problems which are difficult to overcome hinder its practical application $[2,3]$, such as the ablation in the track, the stability of the launching process, the electrical conductivity and the stiffness support [4]. In order to realize the high power of the electromagnetic railgun and can be repeatedly fired, on the one hand, people are exploring the structural form of the composite track, for example, the excellent physical properties of carbon nanotube reinforced copper matrix composites make it a suitable composite material for composite track [5], in addition, $\mathrm{Ti}_{2} \mathrm{AlN}$ is a very comprehensive performance of the copper-based composite material, can make the material with high strength, high elastic modulus and high conductivity, but also an optional composite material of the launch track [6]. On the other hand, it can provide theoretical basis for the design of the track by calculating the dynamic response and stress distribution of the track and analyzing its stress and deformation [7-11]. In [12], the guide rail is simplified as an elastic foundation beam, and the influence of the length of the guide rail on the resonance velocity is discussed under the assumption that the armature passes through the guide rail at a constant speed. The effects of plasma viscosity and inertia resistance on the armature motion are analyzed by numerical simulation [13]. In [14], the characteristics of the recoil motion of the electromagnetic rail gun are analyzed, and the recoil motion curve under certain conditions is obtained. However, these studies do not take into account the effects of armature forces on the track and the characteristics of the armature's variable accelerating motion, the study on the dynamic response of composite track is rare. When the current flows into the composite guide rail, the armature will be subjected to a strong thrust and accelerates the motion along the guide rail. From the distribution of electromagnetic fields between the composite track and the current intensity of the armature, the thrust of the armature is obtained, and the equation of motion of the armature is obtained. By calculating the repulsive force of the interaction between the guide rails and the expansion of the armature caused by the Joule heating, the value of the moving load and its variation law can be obtained. Considering the 
structure of the electromagnetic gun launcher, the control equation of the composite track simplified as the elastic foundation beam is transformed, and the dynamic response equation of the composite track is obtained, on this basis, the expressions of the dynamic moment and dynamic stress of composite track are obtained. By an example, the influence of the material ratio of the composite track and the parameters of the access voltage on the dynamic stress of the composite track is analyzed, which provides the theoretical basis for the design of the composite track.

1. Control Equation of Composite Track. As illustrated in Fig. 1, when an electromagnetic railgun is fired, the high current is charged into the track, the current forms a strong magnetic field between the tracks, the armature sliding along the track at high speed under the action of thrust, at the same time, the track is subject to repulsion, the thermal expansion of the armature and rail due to Joule heat will also be limited, thus, the armature will give a pressure to the track. Considering the effect of the external structure of the track on the track, the composite track at the launching state can be simplified to an elastic foundation beam, as shown in Fig. 2. The intensity of repulsive forces between tracks is $q_{1}(t)$, the pressure of the armature on the track is $P(t), l(t)$ is the distance of the $t$ moment armature along the track, and $a$ is the length of the armature along the track. The dynamic governing equation of the composite track is [15]

$$
\left(E_{1} I_{1}+E_{2} I_{2}\right) \frac{\partial^{4} w(y, t)}{\partial y^{4}}+k w(y, t)+\bar{\rho} h d \frac{\partial^{2} w(y, t)}{\partial t^{2}}=q(y, t),
$$

where $w(y, t)$ is the deflection of the track, $k$ is the elastic coefficient of the foundation, $\bar{\rho}$ is the average mass density of the composite track, $E_{1} I_{1}$ is the flexural rigidity of the basic track, $E_{2} I_{2}$ is the flexural rigidity of the composite layer, $E_{1}$ and $E_{2}$ are, respectively, the elastic modulus of the base material and the composite layer material, $h=h_{1}+h_{2}$ is the thickness of composite track, $h_{1}$ and $h_{2}$ are, respectively, the thickness of the basic track and composite layer track, $d$ is the height of the track, $q(y, t)$ is the load acting on the inside of the track, $q(y, t)=q_{1}(t)[1-H(y-l(t))]+q_{2}(t) H\left[a^{2}-(y-l(t))^{2}\right]$, $q_{2}(t)=P(t) / a$ is the average pressure concentration of the armature acting on the track, and $H(x-l(t))$ and $H\left[a^{2}-(x-l(t))^{2}\right]$ are Heaviside step functions.

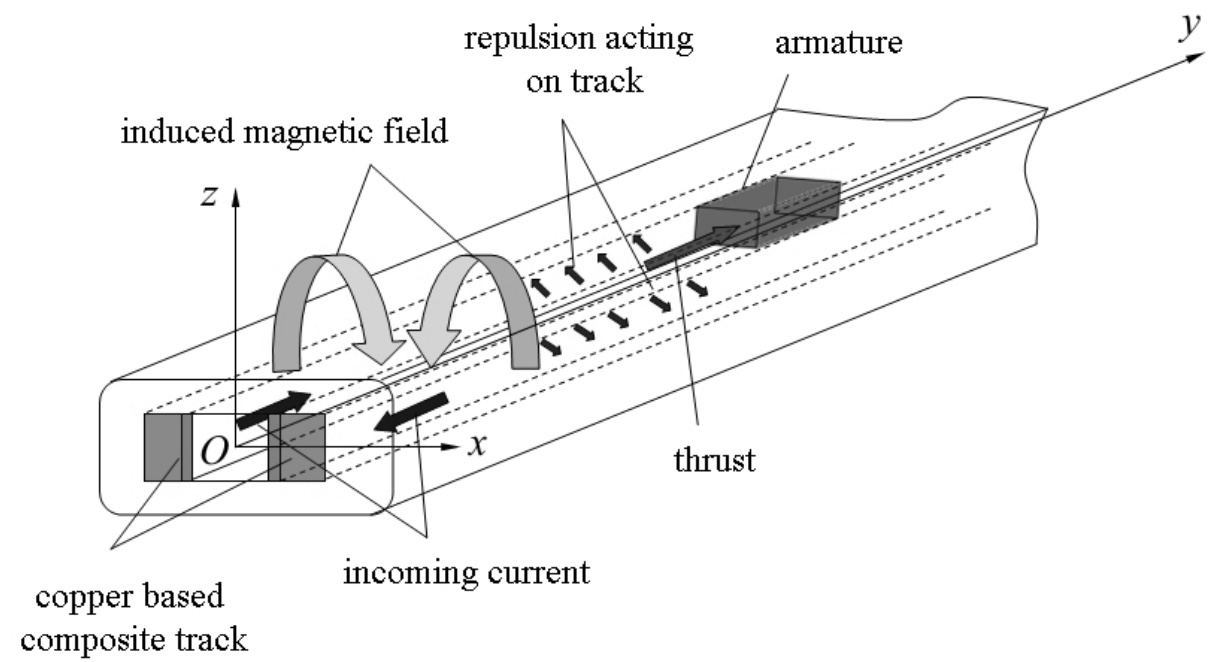

Fig. 1. Schematic of composite rail launcher. 


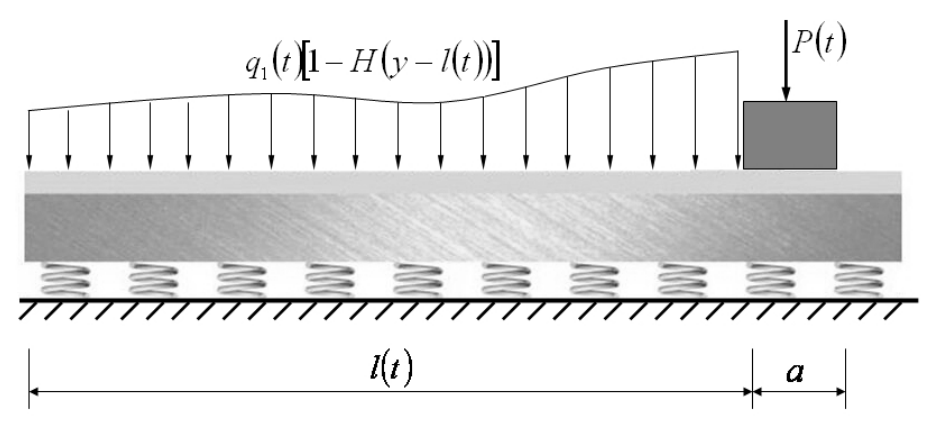

Fig. 2. Schematic of the load state of the composite track.

2. Dynamic Response of Composite Track. By Eq. (1), the application of twodimensional Fourier transform and inverse transform can be used to get the deflection of the rail is as follows:

$$
w(y, t)=\frac{1}{4 \pi^{2}} \int_{-\infty}^{\infty} \int_{-\infty}^{\infty} \frac{\bar{q}(\alpha, \beta) e^{j(\alpha y+\beta t)} d \alpha d \beta}{\left(E_{1} I_{1}+E_{2} I_{2}\right) \alpha^{4}+k-\bar{\rho} h d \beta^{2}},
$$

where

$$
\bar{q}(\alpha, \beta)=\int_{-\infty}^{\infty} \int_{-\infty}^{\infty} q_{1}(t)[1-H(y-l(t))] H+q_{2}(t) H\left[a^{2}-(y-l(t))^{2}\right] e^{-j(\alpha y+\beta t)} d y d t,
$$

$\alpha$ and $\beta$ are integral variables, and $j=\sqrt{-1}$ is a pure imaginary. Formula (3) is introduced into Eq. (2), and the deflection expression of the rail along the composite track is obtained

$$
\begin{aligned}
& w(y, t)=\frac{1}{4 \pi^{2}} \int_{-\infty}^{\infty} \int_{0}^{t} \frac{q_{1}(\tau) e^{j \alpha(y-l(\tau))} d \tau d \alpha}{\left(E_{1} I_{1}+E_{2} I_{2}\right) \alpha^{4}+k-\bar{\rho} h d(l(\tau))^{2}}+ \\
& +\frac{1}{2 \pi^{2}} \int_{-\infty}^{\infty} \frac{\sin (\alpha a) q_{2}(t) e^{j a(y-l(t))} d \alpha}{\left[\left(E_{1} I_{1}+E_{2} I_{2}\right) \alpha^{4}+k-\bar{\rho} h d(l(t))^{2}\right] \alpha a} .
\end{aligned}
$$

The average concentration of the repulsive force of the interaction between the two tracks is [8]

$$
q_{1}(t)=-\frac{\mu_{0} i^{2}(t)}{8 \pi l(t)}\left[\frac{\sqrt{l^{2}(t)+\left(h_{2}+b\right)^{2}}}{h_{2}+b}+1\right]
$$

where $i(t)$ is the current intensity of the incoming track base layer and $b$ is the distance between tracks.

Assuming that both sides of the track limit the thermal expansion of the armature, the average temperature along the $z$ direction is used as the basis for calculating the armature strain, the average concentration of the load on the track is

$$
q_{2}(t)=\frac{Q(t) d^{2} E_{a} \alpha_{T}}{24}\left[\frac{d}{\lambda_{a}}+\frac{6}{\alpha_{a}}\right]
$$


where $Q(t)=i^{2}(t) /\left(h_{1}^{2} d^{2} \sigma_{a}\right)$ is the Joule heat source power density of armature, $E_{a}$ is the elastic modulus of the armature, $\alpha_{T}$ is the coefficient of thermal expansion of the armature, $\lambda_{a}$ is the thermal conductivity of the armature, and $\alpha_{a}$ is the thermal diffusion coefficient of the armature. The formula (5) and (6) are introduced into Eq. (4), and the dynamic response of the track can be obtained after integration.

3. The Dynamic Stress of Composite Track. Figure 3 is a cross-sectional view of one side composite track, $z^{\prime}$ is the neutral axis of the cross section, $c$ is the distance from the inner surface of the track to the neutral axis. According to the equilibrium equation of the section and the properties of the planar graph, the formula of $c$ is obtained as follows:

$$
c=\frac{E_{2} h_{2}^{2}-E_{1} h_{1}^{2}+2 E_{1} h h_{1}}{2\left(E_{2} h_{2}+E_{1} h_{1}\right)} .
$$

The approximate differential equation of the deflection curve of the composite track is

$$
\frac{d^{2} w}{d y^{2}}=\frac{M(y)}{E_{1} I_{1}+E_{2} I_{2}} .
$$

If the influence of rail shear force is neglected, the track is in the pure bending state, the normal stress on the cross section of the track is

$$
\left\{\begin{array}{l}
\sigma_{y 1}=\frac{E_{1} M(y) x^{\prime}}{E_{1} I_{1}+E_{2} I_{2}}, \\
\sigma_{y 2}=\frac{E_{2} M(y) x^{\prime}}{E_{1} I_{1}+E_{2} I_{2}},
\end{array}\right.
$$

where $I_{1}$ and $I_{2}$ are, respectively, the inertia moment of the base layer and track composite layer to the neutral axis, and $\sigma_{y 1}$ and $\sigma_{y 2}$ are, respectively, the normal stresses in the $y$-axis direction on the cross section of the track base and the track composite layer, At this point, the normal stress on the cross section of the composite track is obtained by Eqs. (4), (8), and (9).

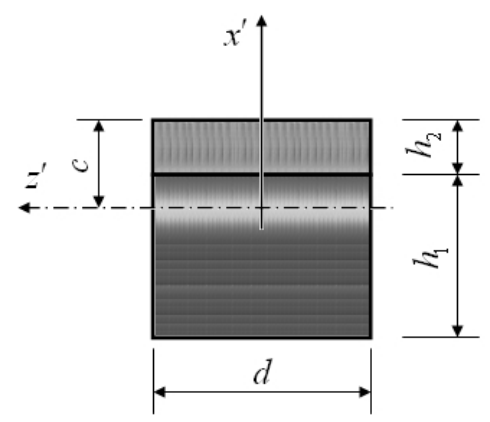

Fig. 3. The cross section of composite track.

\section{Analysis of Examples.}

4.1. Theoretical Analysis. The composite track base layer is made of copper, and the composite layer is made of steel, the track length is $2 \mathrm{~m}$, the thickness of the track base layer is $h_{1}=30 \mathrm{~mm}$, the thickness of the composite layer is $h_{2}=10 \mathrm{~mm}$, the distance between the two tracks is $b=20 \mathrm{~mm}$, the mass density of copper is $\rho_{1}=8900 \mathrm{~kg} / \mathrm{m}^{3}$, the 
elastic modulus is $E_{1}=115 \mathrm{GPa}$, Poisson's ratio is $v_{1}=0.33$, and conductivity is $\sigma_{1}=$ $=5.88 \cdot 10^{7}(\Omega \cdot \mathrm{m})^{-1}$. The mass density of steel is $\rho_{2}=7850 \mathrm{~kg} / \mathrm{m}^{3}$, the elastic modulus is $E_{2}=210 \mathrm{GPa}$, Poisson's ratio is $v_{2}=0.3$, conductivity is $\sigma_{2}=9.93 \cdot 10^{6}(\Omega \cdot \mathrm{m})^{-1}$, and the initial resistance is $R_{0}=0.01 \Omega$. The length of the aluminum armature in the direction of the guide rail is $a=20 \mathrm{~mm}$, the thickness and height of armature both are $20 \mathrm{~mm}$, conductivity is $\sigma_{a}=3.6 \cdot 10^{7}(\Omega \cdot \mathrm{m})^{-1}$, the mass density is $\rho_{a}=2700 \mathrm{~kg} / \mathrm{m}^{3}$, the thermal conductivity is $\lambda_{a}=237 \mathrm{~W} /\left(\mathrm{m} \cdot{ }^{\circ} \mathrm{C}\right)$, the expansion coefficient is $\alpha_{T}=2.35 \cdot 10^{-5}{ }^{\circ} \mathrm{C}^{-1}$, and the exothermic surface coefficient is $\alpha_{a}=500 \mathrm{~W} /\left(\mathrm{m} \cdot{ }^{\circ} \mathrm{C}\right)$. The system discharge current is $i_{0}=50 \mathrm{kA}$, the vacuum permeability is $\mu_{0}=1.256 \cdot 10^{-6} \mathrm{H} / \mathrm{m}$, and the elastic coefficient of the foundation is $k=10 \mathrm{GPa}$.

When the thickness ratio of the track is $h_{2} / h_{1}=1: 3$, the normal stress $\sigma_{y}$ of $x^{\prime}=$ $=-22.6,7.4$, and $17.4 \mathrm{~mm}$ on the section of $y=0.8 \mathrm{~m}$ is changed with the armature moving distance $l(t)$, as shown in Fig. 4 , it can be calculated by Eq. (5) at this time that $c=17.4 \mathrm{~mm}$, thus, the three curves are the normal stress on the outer surface of the track, the normal stress at the interface of the track, and the normal stress on the inner surface of the track. It can be seen that when the armature passes through the $y=0.8 \mathrm{~m}$, the normal stress reaches the maximum value, the stress on the inner surface of the track is the largest, and the extreme value is the tensile stress, and the stress of the outer surface of the track is the compressive stress, the value is smaller than the absolute value of the inner surface stress, and the stress at the interface of the track is relatively small, because it is closer to the neutral axis. This explains why the inner surface of the rail is more vulnerable to damage.

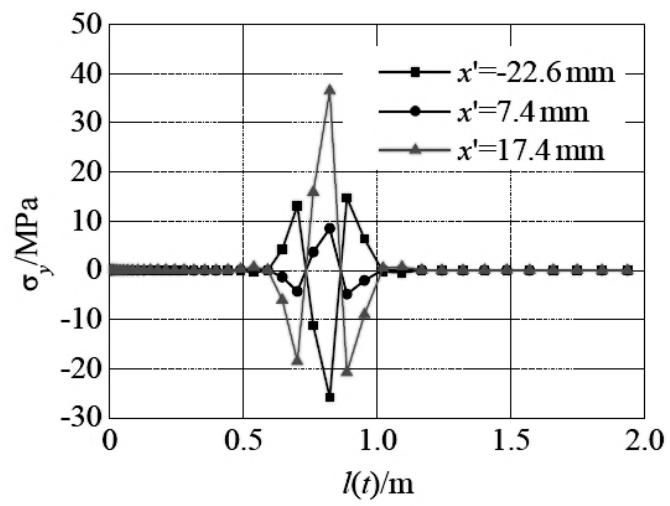

Fig. 4. Variation of $\sigma_{y}$ at $0.8 \mathrm{~m}$ of track versus the armature moving distance $l(t)$.

The curves of the inner surface normal stress in $y$ direction with the moving distance of the armature is shown in Fig. 5, when the track thickness is $h=40 \mathrm{~mm}$, and the track thickness ratio is different. It can be seen that with the increase of the thickness of the composite layer, the normal stress increases, the maximum value of the stress is increased by about $31 \%$. The curves of the inner surface normal stress in the $y$ direction at $y=0.8$, 1.0 , and $1.2 \mathrm{~m}$ with the moving distance of the armature is shown in Fig. 6, when the track thickness ratio is $h_{2} / h_{1}=1: 7$, as can be seen in the Fig. 6 , when the armature through the location, the stress of the location appears extreme value, and in the position earlier or later when the stress is negative, when the armature slides in other locations on the track, the position of the analysis will also have an impact, but the absolute value of the stress caused by it than the armature through this position caused by the stress is much smaller.

The curves of the inner surface normal stress in $y$ direction at $y=0.8 \mathrm{~m}$ with the moving distance of the armature is shown in Fig. 7, when the track thickness ratio is 


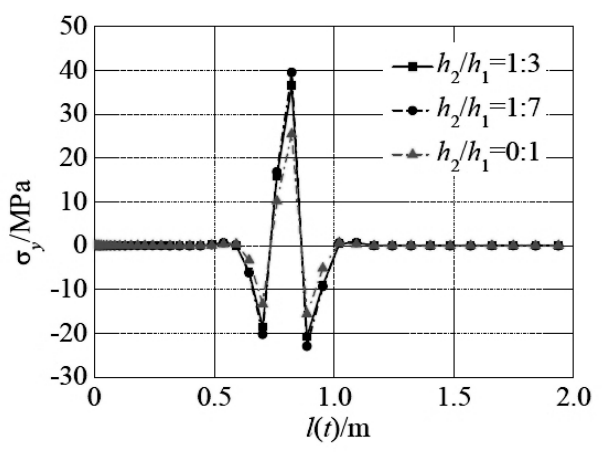

Fig. 5

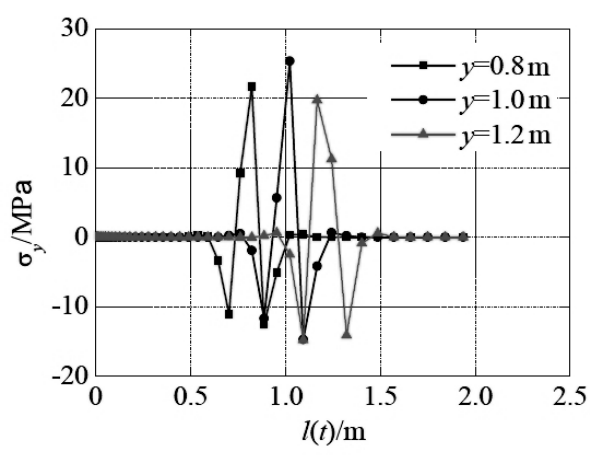

Fig. 6

Fig. 5. The curves of the inner surface stress $\sigma_{y}$ with $l(t)$ changes when the track thickness ratio is different.

Fig. 6. The relationship between the inner surface stress $\sigma_{y}$ and $l(t)$ at the different position of the track.

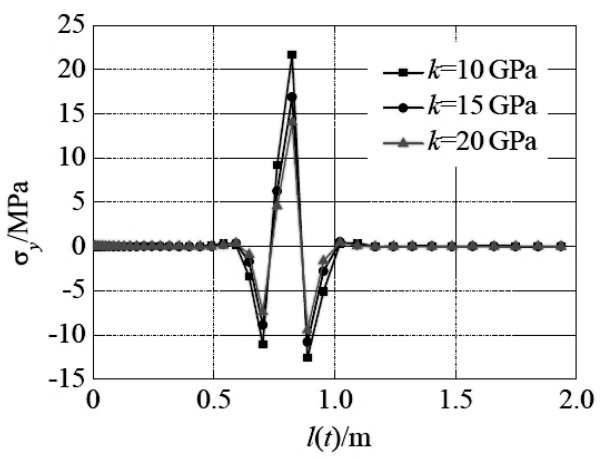

Fig. 7

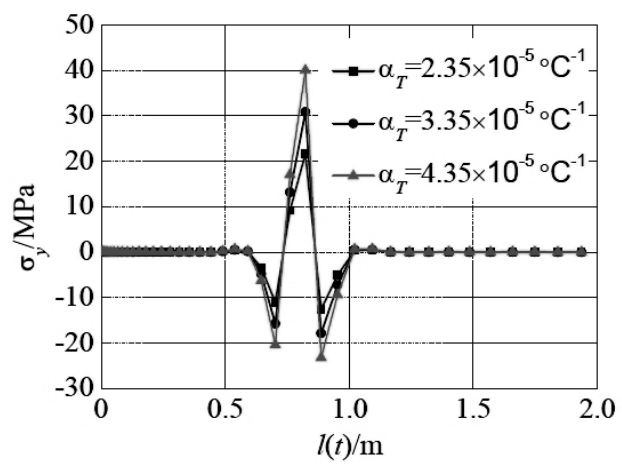

Fig. 8

Fig. 7. The curves of the internal surface stress $\sigma_{y}$ with $l(t)$ changes when the elasticity coefficient of the foundation is different.

Fig. 8. The curves of the inner surface stress $\sigma_{y}$ with $l(t)$ changes when the thermal expansion coefficient of the armature material is different.

$h_{2} / h_{1}=1: 7$, and the elastic coefficients of foundation are $k=10,15$, and $20 \mathrm{GPa}$, respectively, it can be seen that increasing the external constraint of the track, that is, increasing the elasticity coefficient of the foundation can effectively reduce the stress peak of the inner surface of the track.

The curves of the inner surface normal stress in the $y$ direction at $y=0.8 \mathrm{~m}$ with the moving distance of the armature is shown in Fig. 8, when the track thickness ratio is $h_{2} / h_{1}=$ $=1: 7$, and the thermal expansion coefficient of armature material are $\alpha_{T}=2.35 \cdot 10^{-5}$, $3.35 \cdot 10^{-5}$, and $4.35 \cdot 10^{-5}{ }^{\circ} \mathrm{C}^{-1}$, respectively, as can be seen from the Fig. 8, with the increase of the thermal expansion coefficient of the armature material, the stress on the inner surface of the track is increased, the maximum value of the stress is increased by about $45 \%$, which can be understood that the temperature rise of the armature is the same under the same launching conditions, however, due to the increase of the coefficient of thermal expansion of the armature, the expansion of the armature along the $y$-direction increases, which results in an increase in the force acting on the track, therefore, reducing the thermal expansion coefficient of the armature material can reduce the stress of the railgun at launching state. 
4.2. Numerical Simulation Analysis. In order to analyze the stress state of the composite track from different angles, the Comsol software is used to simulate the multiphysics field of the railgun. The geometric parameters and material properties are consistent with the theoretical calculation.

Figure 9 shows the distribution of the normal stress $\sigma_{y}$ of the track when the armature is moved to $y=1 \mathrm{~m}$, it is shown that the stress on the orbit around the armature is larger than that of the other position, the distribution law is consistent with the theoretical results, but the absolute value of the stress peak is larger than the theoretical calculation result, mainly because the theoretical calculation does not take into account the "rigid" effect of the armature on the track.

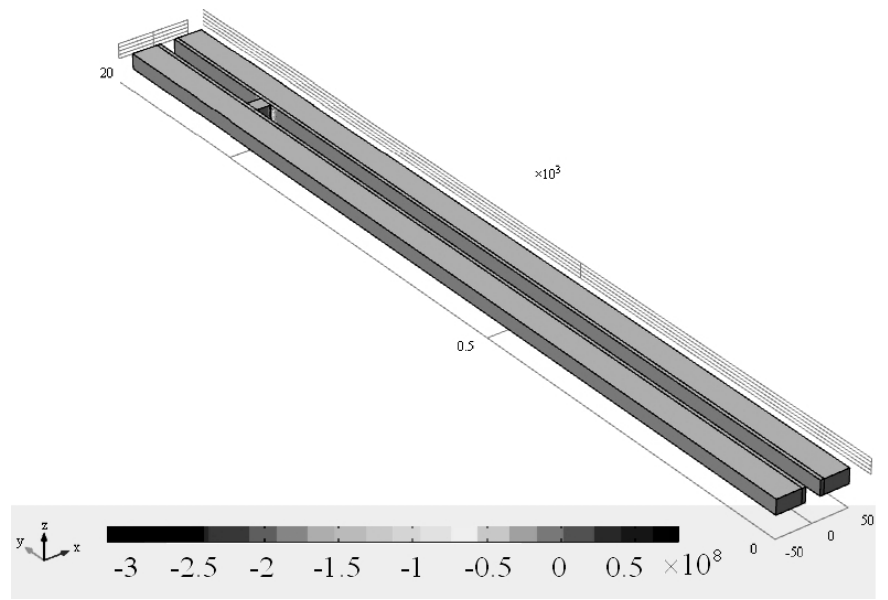

Fig. 9. Nephogram of stress $\sigma_{y}$ distribution at launching state.

Figure 10 shows the normal stress $\sigma_{y}$ distribution on the different longitudinal faces $x=-20,-30$, and $-40 \mathrm{~mm}$ of the track when the armature moves to $y=1 \mathrm{~m}$. Figure 10 shows that the more close to the inner surface of the track, the more significant the role of armature, in comparison with Fig. 4, it can be found that the closer the position to the outer surface of the track, the closer the simulation results are to the theoretical results, because the "rigid " effect of the armature on the track is weakened as the depth increases.

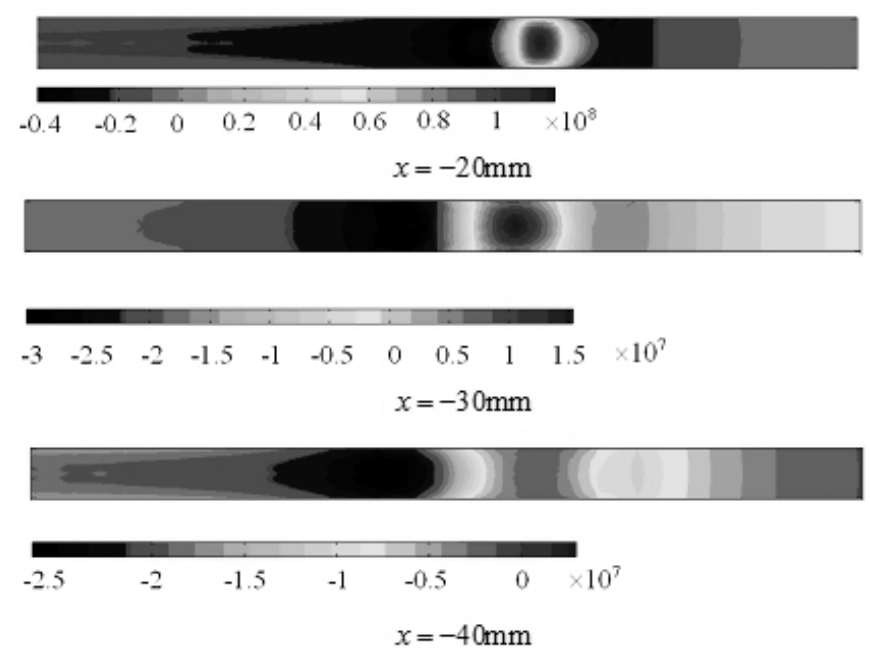

Fig. 10. Nephogram of stress $\sigma_{y}$ distribution on different surfaces of the base layer. 
Figure 11 shows the distribution of the temperature of the armature when the armature is moved to $y=1 \mathrm{~m}$, it can be seen that the temperature at the end of the armature in the contact surface between the armature and the track is the highest, and the middle position is lower, the maximum temperature is about three times the minimum internal temperature, this is due to the uneven distribution of current density caused by current flow effect.

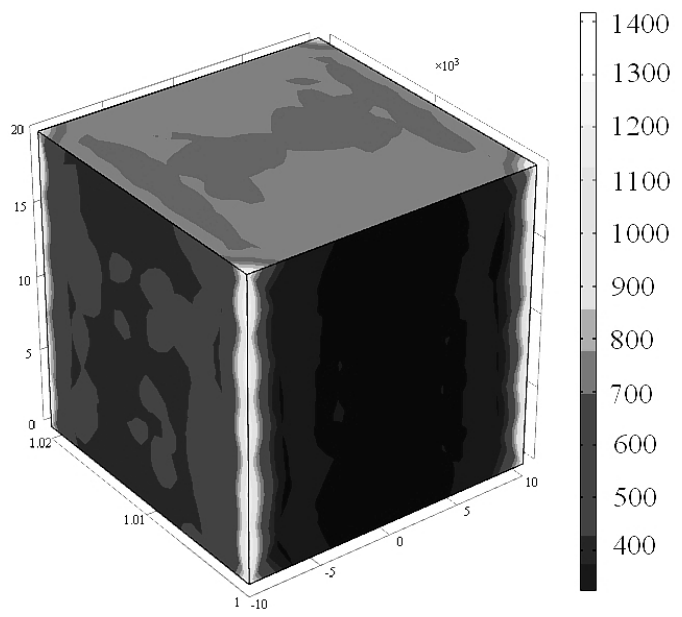

Fig. 11. Temperature nephogram of the armature.

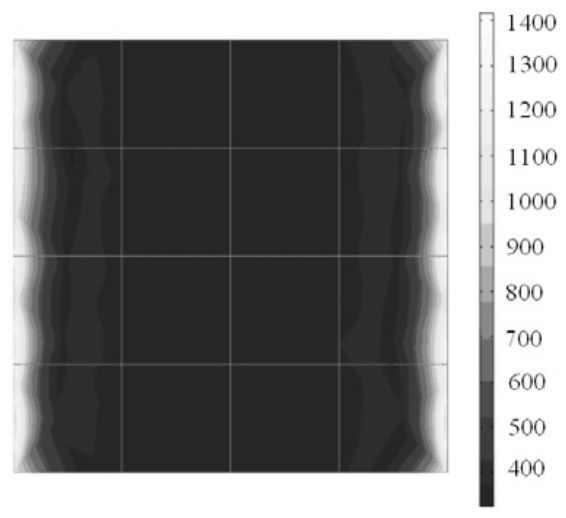

$y=1000 \mathrm{~mm}$

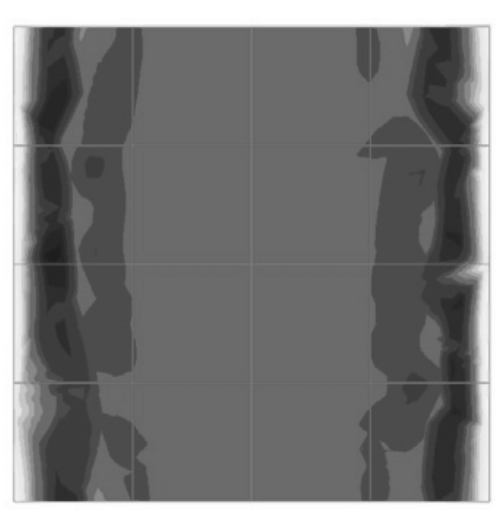

$y=1010 \mathrm{~mm}$

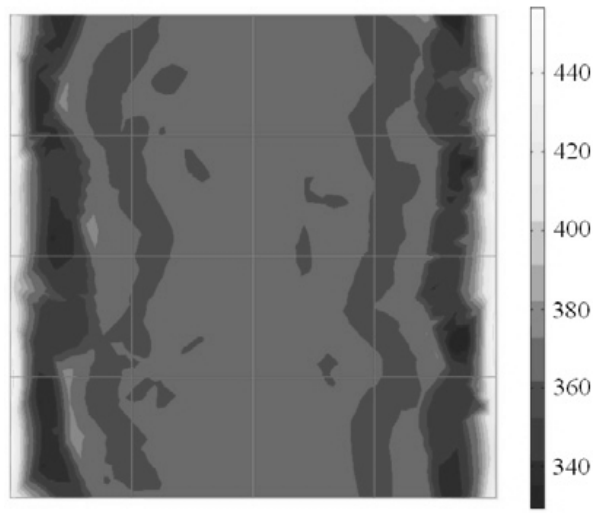

$y=1005 \mathrm{~mm}$

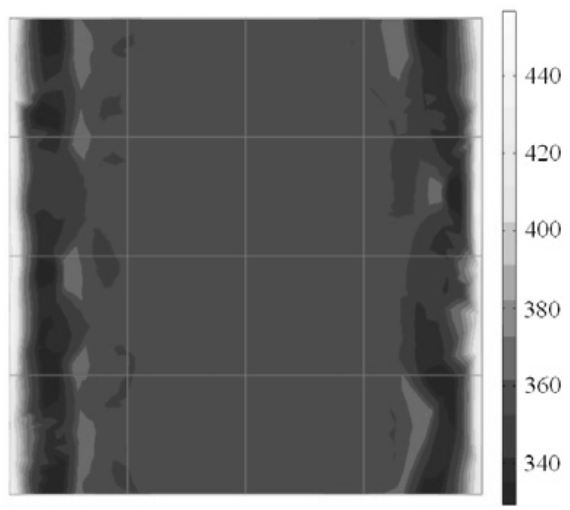

$y=1015 \mathrm{~mm}$

Fig. 12. Temperature nephogram in different faces of the armature. 
Figure 12 shows the temperature distribution on the armature along the $y$-direction cross sections $y=1000,1005,1010$, and $1015 \mathrm{~mm}$, respectively, it can be seen from the observation that the temperature at the adjacent launch end is higher and the temperature on the outlet side is relatively low, the maximum temperature is close to $1400^{\circ} \mathrm{C}$, which causes the local thermal expansion of armature to increase, resulting in the increase of local pressure on the track, causing the track local stress to rise sharply, resulting in track damage. This is mainly due to the roundabout flow phenomenon, and the current density distribution in Fig. 13 illustrates this. The distribution of the current density is uniform at the front of the launching end of the electromagnetic gun, in the vicinity of the armature, the distribution of current density is uneven, especially in the vicinity of the interface of the track and armature, and the concentration of current density is apparent.

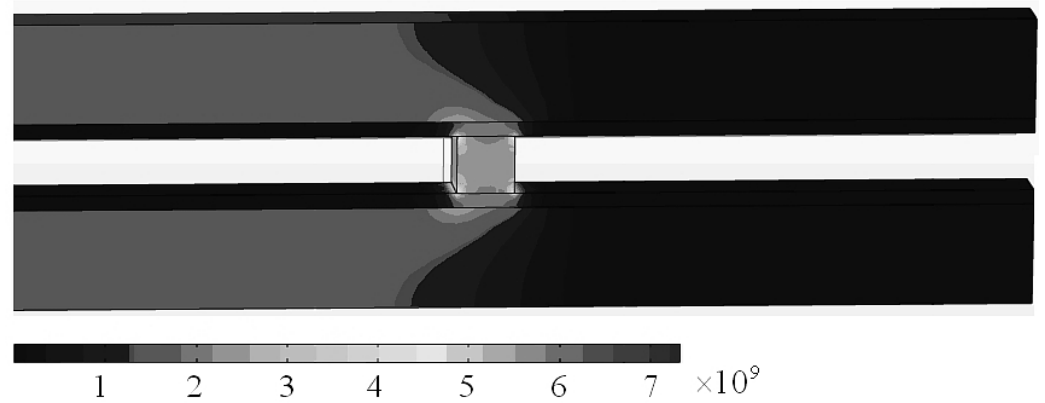

Fig. 13. The current density nephogram of armature and track at launching state.

Conclusions. Theoretical and numerical results show that the thermal expansion of the armature caused by the electrothermal effect is the main factor leading to the deformation and failure of the track, the roundabout flow phenomenon causes the armature current density to be concentrated when the current flow from the track to the armature, so that the pressure in the vicinity of the end point of the armature and the rail contact surface is increased, resulting in a sharp increase in the stress on the inner surface of the local rail.

The calculation results show that when the thermal expansion coefficient of the armature increases by $86 \%$, the tensile stress of the inner surface of the rail is increased by $45 \%$, therefore, reducing the coefficient of thermal expansion and controlling the expansion of the armature is an effective way to minimize the damage of the track under the same launching conditions. The thickness of the composite layer and the stiffness of the external support of the track influence the dynamic stress of the track when the stiffness of the external support is doubled, the stress on the inner surface can be reduced by about $33 \%$.

The material with high conductivity, high strength, and low thermal expansion coefficient can be used to improve the overall strength of the composite track, at the same time, taking into account the rail conductivity and the overall stiffness of the track, to select a reasonable proportion of the thickness of the composite layer.

Acknowledgments. This work is supported by Natural Science Foundation of Hebei Province (A2015203086).

1. H. D. Fair, "The past, present, and future of electromagnetic launch technology and the IEEE international EML symposia," IEEE T. Plasma Sci., 41, No. 5, 1024-1027 (2013).

2. H. D. Fair, "Advances in electromagnetic launch science andtechnology and its applications," IEEE T. Magn., 44, No. 1, 225-230 (2009).

3. I. R. Mcnab and F. C. Beach, "Naval railguns," IEEE T. Magn., 43, No. 1, 463-468 (2007). 
4. B. Lei, R. G. Zhu, Q. Zhang, et al., "Production features and research status of gouging in electromagnetic railgun," Ordn. Mater. Sci. Eng., 34, No. 3, 76-80 (2011). (in Chinese)

5. K. H. W. Kenneth, Z. A. Martin, L. H. Jeffery, et al., "The effect of carbon nanotube aspect ratio and loading on the elastic modulus of electrospun poly(vinyl alcohol)carbon nanotube hybrid fibers," Carbon, 47, No. 11, 2571-2578 (2009).

6. W. Wang, Z. Zhan, Q. Tang, et al., "Interfacial reaction and properties of $\mathrm{Ti}_{2} \mathrm{AlN}-$ $\mathrm{La}_{2} \mathrm{O}_{3} / \mathrm{Cu}$ composite," Rare Metal Mater. Eng., 44, No. 5, 1177-1180 (2015). (in Chinese)

7. C. Fan and W. Wang, "Review on the electromagnetic railgun," J. Yanshan Univ., 31, No. 5, 377-386 (2007).

8. Z. G. Tian, X. Z. Bai, and Y. Yang, "Dynamic response of rail during launch process of electromagnetic rail," J. Vib. Shock, 31, No. 2, 10-14 (2012).

9. C. Wei, Y. Xu, and Y. Y. Ma, "Study on damage of composite material barrel under transient impact load," J. Ordn. Equip. Eng., 37, No. 7, 131-136 (2016). (in Chinese)

10. Z. G. Tian, X. Y. Meng, X. Y. An, et al., "Dynamic response of composite rail during launch process of electromagnetic railgun," ACTA Armamentarii, 38, No. 4, 651-657 (2017). (in Chinese)

11. Z. G. Tian and X. Y. An, "Multi physical field coupling analysis of composite electromagnetic track," J. Gun Launch \& Control, 38, No. 3, 1-6 (2017). (in Chinese)

12. J. T. Tzeng and W. Sun, "Dynamic response of cantilevered rail guns attributed to projectile/gun interaction-theory," IEEE T. Magn., 43, No. 1, 207-213 (2007).

13. Y. Yang and J. X. Wang, "Numerical modeling and analysis on EM gun firing principle," J. Gun Launch \& Control, 4, 9-13 (2008). (in Chinese)

14. J. Shi and B. M. Li, "Research on recoil process of electromagnetic railgun," ACTA Armamentarii, 36, No. 2, 227-233 (2015). (in Chinese)

15. W. P. Xie and G. Wang, "Analysis of dynamic characteristics of rail system induced by moving load," J. Zhengzhou Univ., 24, No. 1, 24-27 (2003). (in Chinese)

Received 05. 03. 2018 